\title{
Different methods for target volume delineation of glandular breast tissue following breast-conserving surgery in breast cancer: A comparative study
}

\author{
MIN XU ${ }^{1,2}$, JIANBIN LI $^{2}$, SHANSHAN LIU ${ }^{2}$, SUZHEN WANG $^{2}$, WEI WANG ${ }^{2}$, FENGXIANG LI $^{2}$, \\ TONGHAI LIU ${ }^{2}$ and JINMING YU ${ }^{1,2}$ \\ ${ }^{1}$ Shandong University School of Medicine; ${ }^{2}$ Department of Radiation Oncology, \\ Shandong Cancer Hospital and Institute, Jinan, Shandong, P.R. China
}

Received July 25, 2014; Accepted February 23, 2015

DOI: $10.3892 / \mathrm{ol} .2015 .3358$

\begin{abstract}
The present study aimed to investigate an optimal and feasible method for delineating the target volume of glandular breast tissue following breast-conserving surgery. A total of 15 patients who underwent radiotherapy following breast-conserving surgery were recruited into the study. Clinical target volume was delineated by the following three methods based on computed tomography (CT): Anatomical landmarks (CTVan), breast palpation (CTVpa) and CT scan images (CTVgl). The target volume, degree of inclusion (DI) and conformal index (CI) defined by these methods were compared. The difference was significant between CTVan and CTVgl, and CTVpa and CTVgl (P<0.0001). The CI between CTVan and CTVpa was $0.644 \pm 0.122$, significantly higher than that between CTVan and CTVgl (0.264 \pm 0.108 ; $\mathrm{P}<0.0001)$ or between CTVpa and CTVgl (0.328 \pm 0.115 ; $\mathrm{P}<0.0001)$. The DI of CTVpa in CTVan was $0.890 \pm 0.08$ and the opposite was $0.709 \pm 0.144$, while that of DI of CTVgl in CTVan or CTVpa was $0.994 \pm 0.005$ and $0.989 \pm 0.008$, respectively. The boundary difference between CTVan and CTVpa was $3.35 \pm 7.23,5.57 \pm 13.37,1.75 \pm 11.62$ and $11.25 \pm 4.07 \mathrm{~mm}$ for the medial, lateral, cephalic and caudal boundaries, respectively. A significant difference was observed in the target volume of the breast defined by the three methods. The target volume defined by CTVgl was significantly smaller than that identified by the other two methods. Overall, the combination of palpation marks and anatomical landmarks to define the contouring scope of the breast was indicated to be a relatively rational method for delineating the target volume of the breast.
\end{abstract}

Correspondence to: Professor Jinming Yu, Department of Radiation Oncology, Shandong Cancer Hospital and Institute, 440 Jiyan Road, Jinan, Shandong 250117, P.R. China

E-mail: yujinmingsd@126.com

Key words: clinical target volume of breast, target delineation, anatomical landmark, palpation mark, computed tomography image

\section{Introduction}

Randomized clinical studies and meta-analyses (1-3) have provided compelling evidence that a combination of post-operative radiotherapy and breast-conserving surgery reduces the local recurrence of breast cancer. Therefore, post-operative radiation is an indispensable therapy integrated into the standard therapeutic scheme for breast cancer patients, with the exception of those patients aged $>70$ years and those with early-stage cancer with an estrogen receptor-positive, prolactin receptor-positive and human epidermal growth factor receptor-2-negative genotype who could adopt endocrine therapy only after surgery. Currently, boost irradiation for the whole breast following breast-conserving surgery has been regarded as a classic radiotherapy scheme (4-6). Although accelerated partial breast irradiation has been demonstrated to be superior in terms of less adverse reactions and a shortened treatment period compared with conventional irradiation, and has been widely accepted in clinical practice in recent years, it is mainly adopted for low-risk patients with breast cancer at an early stage (7). Whole-breast irradiation remains an irreplaceable therapy following breast-conserving surgery for high-risk patients, however, the hypofractionated radiotherapy for the whole breast remains under evaluation. Therefore, determination of the target volume of the breast is considered critical for post-operative irradiation therapy. Currently, conformal modulated radiotherapy is the mainstream treatment for breast cancer $(4,8,9)$. The post-operative intensity-modulated radiotherapy for breast cancer is usually plotted based on the image from computed tomography (CT) scan, and delineation of the target volume is based on the CT-simulated location scan image; however, there is lack of clearly defined boundaries between mammary gland and non-gland adipose tissue in the images of the CT scan (10). It remains to be elucidated whether the target volume of glandular breast tissue delineated by the CT scan is sufficient for radiotherapy or not. Given the limitation in delineating glandular breast tissue by the CT scan, the anatomical or surface landmarks would be usually considered as a reference tool in clinical practice. Delineation of target volume based on the surface 
marks or anatomical landmarks displayed by the image from the CT scan has been used for conventional tangential irradiation for the whole breast, and ensures that the gland tissue does not miss receiving radiation $(11,12)$. However, this method cannot be individualized for each patient, as the position, size, shape and structure of the breast of each patient are different, with consequent overexposure or underexposure to the radiation. Underexposure may lead to missing the target and the risk of recurrence, and overexposure may result in severe acute and chronic skin reactions (12-14). In addition, other organs, such as the lung and heart, may be irradiated. Therefore, determination of the target volume by palpation is comparatively accurate and individualized. Based on the physical examination and palpation, Bentel et al (11) placed a metal wire around the breast, which was then used as a reference to delineate the target. They found that the target volume differed in $65 \%$ of breasts tested; $30 \%$ exhibited a difference in the medial boundary and $56 \%$ in the lateral boundary. Subsequently, this method has been continuously used by researchers to delineate the target volume of the whole breast. However, there is a lack of a standardized delineation method for delineating the target volume of the whole breast. Therefore, in the current study, a comparison was performed between the three aforementioned delineation methods in order to find out an optimal and feasible method for clinical practice.

\section{Materials and methods}

Inclusion and exclusion criteria. The current study was approved by the Ethics Committee of Shandong Cancer Hospital and Institute (Jinan, China), and written informed consent was obtained from each participant. A total of 15 patients with breast cancer who underwent post-operative radiotherapy between May 2012 and November 2012 were enrolled into the present study. The inclusion criteria were as follows: An age of between 20 and 60 years old; local resection with extended scope for breast tumor removal, and breast tumor determined as stage T1N0M0 or T2N0M0 post-operatively with a diameter ranging between 0.5 and $3.0 \mathrm{~cm}$; and no medical history of breast radiotherapy. The exclusion criteria were as follows: An age of $>60$ years; radical mastectomy or modified radical surgery, or quadrant resection for breast tumor removal; a breast tumor with a diameter of $>3.0 \mathrm{~cm}$; and a medical history of breast trauma or breast radiotherapy.

CT simulation location method. First, the patients were secured with a bracket. The patients were placed in a supine position and their bilateral arms were opened and lifted. The bracket was adjusted to ensure the most comfortable position for patients and the affected breast was fully exposed. The edge of the mammary gland was detected by palpation, along which a metal wire was placed and fixed on the skin surface. Metal tags were placed $10 \mathrm{~mm}$ beneath the breast folds, at the axillary midline and the posterior axillary line of the same side, respectively, as laser-positioning marks. A continuous spiral CT scan with a 3-mm layer thickness was performed for each patient under free-breathing conditions, from the cricothyroid membrane to the bottom of the lung, with a 5-cm caudal extension. All images were sent to the Eclipse ${ }^{\mathrm{TM}}$ treatment planning system (Varian Medical Systems, Inc., Palo Alto, CA, USA).

Delineation of target volume. The target volume was delineated by a senior radiation oncologist with $>5$ years of experience. The radiation oncologist was familiar with the breast anatomy and breast imaging, and was able to skillfully utilize the Eclipse delineation tool.

Methods of delineating target volume. Based on the images from the CT scan, the following three methods were used to delineate the clinical target volume (CTV): CT scan images (CTVgl), anatomical landmarks (CTVan) and breast palpation (CTVpa). The window width of the CT image was set as $400 \mathrm{HU}$ and window level was set as $750 \mathrm{HU}$, so that the contrast image between the mammary gland tissue and the surrounding adipose tissue was relatively clear. While one delineation method was used, the other two were switched off to avoid any potential interference.

To delineate the target volume by surface marks of the breast (CTVan), the anterior boundary was $5 \mathrm{~mm}$ beneath the skin, the posterior boundary was at the anterior edge of the pectoralis major muscle, the cephalic boundary was at the inferior edge of the collarbone, the caudal boundary was $10 \mathrm{~mm}$ beneath the ipsilateral breast folds, the medial boundary was at the parasternal line and the lateral boundary was at the midaxillary line or posterior axillary line (Fig. 1A).

To delineate the target volume by palpation marks of the breast (CTVpa), the cephalic, caudal, medial and lateral boundaries were defined based on the metal wire placed according to palpation. The delineation scope included the external edge of the metal wire. The anterior boundary was $5 \mathrm{~mm}$ beneath the skin and the posterior boundary was at the anterior edge of the pectoralis major muscle (Fig. 1B).

To delineate the target volume by the CT scan images (CTVgl), delineation was performed based on the breast gland tissue displayed by images of the CT scan, excluding the surrounding adipose tissues (Fig. 1C).

Calculation of target volume and comparison of parameters. The CTV was calculated by the Eclipse treatment planning system based on the three different delineation methods. The degree of inclusion (DI) and conformal index (CI) were compared among the three methods.

The DI represents the inclusion of one volume in another volume. The formula for the DI of target $\mathrm{A}$ in target $\mathrm{B}$ is $\mathrm{DIA}=\mathrm{A} \cap \mathrm{B} / \mathrm{A}$, meaning the percentage of overlapping areas (between targets $\mathrm{A}$ and $\mathrm{B}$ ) in target $\mathrm{A}$, while the formula for the DI of target $B$ in target $A$ is $D I B=A \cap B / B(15)$. Assuming target $\mathrm{B}$ as the standard target, target $\mathrm{A}$ was used for radiotherapy, therefore, normal tissue of 1-DIA received unnecessary irradiation. Meanwhile, the target tissue of 1-DIB missed the irradiation received.

The $\mathrm{CI}$ is defined as the ratio of overlapping areas (between targets $\mathrm{A}$ and $\mathrm{B}$ ) to the combination of targets $\mathrm{A}$ and $B(C I=A \cap B / A \cap B)$, representing the similarity between targets $\mathrm{A}$ and $\mathrm{B}$. If two target volumes are identical, $\mathrm{CI}=1$, whereas if two target volumes are not overlapping at all, $\mathrm{CI}=0$ (16). 
Table I. General characteristics of the patients.

\begin{tabular}{|c|c|c|}
\hline \multirow[b]{2}{*}{ Clinical features } & \multicolumn{2}{|c|}{ Patients } \\
\hline & $\mathrm{n}$ & $\%$ \\
\hline \multicolumn{3}{|l|}{ Age range, years } \\
\hline $30-40$ & 6 & 40.0 \\
\hline $40-50$ & 7 & 46.7 \\
\hline $50-60$ & 2 & 13.3 \\
\hline \multicolumn{3}{|l|}{ Breast } \\
\hline Left & 9 & 60.0 \\
\hline Right & 6 & 40.0 \\
\hline \multicolumn{3}{|l|}{ Quadrant } \\
\hline Upper outer & 8 & 53.3 \\
\hline Lower outer & 3 & 20.0 \\
\hline Upper inner & 4 & 26.7 \\
\hline Lower inner & 0 & 0.0 \\
\hline \multicolumn{3}{|l|}{ Size of primary tumor, $\mathrm{cm}$} \\
\hline$\leq 1$ & 3 & 20.0 \\
\hline $1-2$ & 7 & 46.7 \\
\hline$>2$ & 5 & 33.3 \\
\hline \multicolumn{3}{|l|}{ Clinical stage } \\
\hline T1N0M0 & 10 & 66.7 \\
\hline T2N0M0 & 5 & 33.3 \\
\hline \multicolumn{3}{|c|}{ Axillary lymph node removal method } \\
\hline Sentinel lymph node biopsy & 11 & 73.3 \\
\hline Axillary lymphadenectomy & 4 & 26.7 \\
\hline \multicolumn{3}{|l|}{ Post-operative chemotherapy } \\
\hline Yes & 12 & 80.0 \\
\hline No & 3 & 20.0 \\
\hline \multicolumn{3}{|l|}{ Post-operative endocrine therapy } \\
\hline Yes & 3 & 20.0 \\
\hline No & 12 & 80.0 \\
\hline
\end{tabular}

Statistical analysis. Statistical analysis was performed using SPSS 19.0 software (IBM, Armonk, NY, USA). One-way analysis of variance was used for analyzing the differences among the three methods based on the evaluation of the target volume. Paired t-test was used for two-by-two comparison. $\mathrm{P}<0.05$ was considered to indicate a statistically significant difference.

\section{Results}

Patient characteristics. The general characteristics of the patients, including patient age, tumor size and location, and treatment administered, are listed in Table I.

Comparison of target volume. The target volumes defined by CTVan, CTVpa and CTVgl were 792.229 282.246 , $618.331 \pm 295.903$ and $196.825 \pm 117.618 \mathrm{~cm}^{3}$, respectively, with significant differences among the methods $(\mathrm{F}=17.161$; $\mathrm{P}<0.0001)$. Two-by-two comparison indicated that there was no significant difference between CTVan and CTVpa $(\mathrm{t}=3.256$;
A

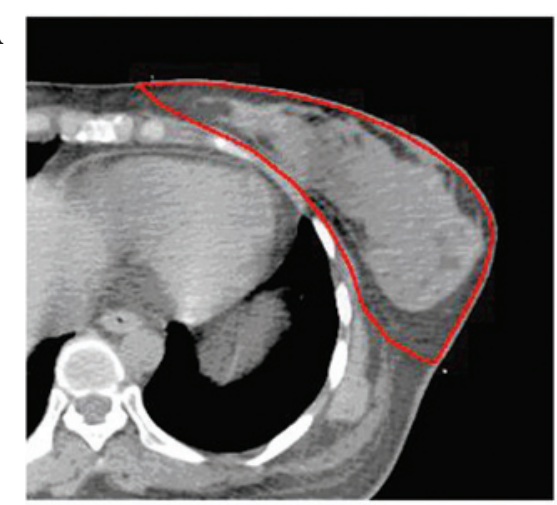

B

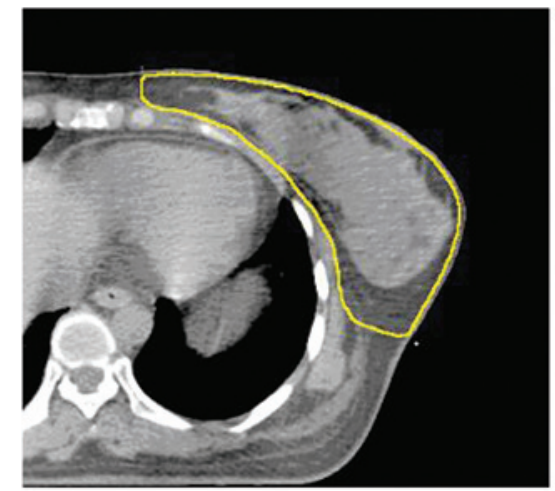

C

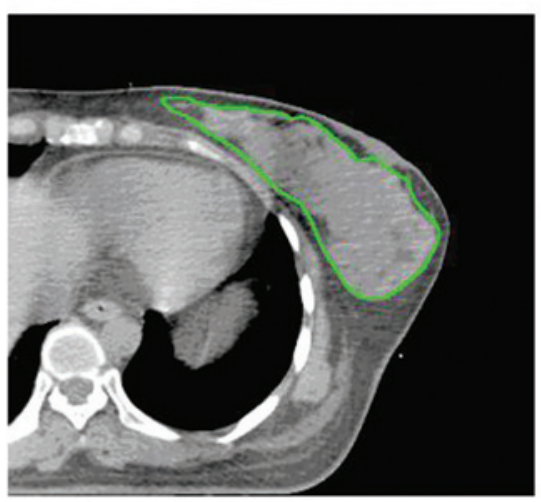

Figure 1. (A) The target volume of the breast delineated according to the anatomical landmarks, depicted as CTVan. (B) The target volume of the breast delineated according to the palpation marks, depicted as CTVpa. (C) The target volume of the breast delineated based on the images of the computed tomograpy scan, depicted as CTVgl. CTV, clinical target volume.

$\mathrm{P}=0.08$ ); whereas there was a significant difference between CTVan and CTVgl $(\mathrm{t}=8.433 ; \mathrm{P}<0.0001)$ and between CTVpa and CTVgl $(\mathrm{t}=6.001 ; \mathrm{P}<0.0001)$.

Comparison of CI. CI1-2 is defined as the CI between CTVan and CTVpa, with a mean of $0.644 \pm 0.122$ (range, 0.490-0.859); CI1-3 is defined as the CI between CTVan and CTVgl, with a mean of $0.264 \pm 0.108$ (range, $0.130-0.423$ ); and CI2-3 is defined as the CI between CTVpa and CTVgl, with a mean of $0.328 \pm 0.115$ (range, 0.151-0.522). There was a significant difference between CI1-2 and CI1-3 ( $\mathrm{t}=14.708 ; \mathrm{P}<0.0001)$ and also between CI1-2 and CI2-3 ( $\mathrm{t}=8.012 ; \mathrm{P}<0.0001)$.

Comparison of DI. The DI of CTVpa in CTVan was 0.890 \pm 0.08 (range, 0.774-0.990), the DI of CTVan in CTVpa was $0.709 \pm 0.144$ (range, 0.492-0.892), the DI of CTVgl in CTVan was 0.994 \pm 0.005 (range, 0.985-1.000), the DI of CTVan 
Table II. Boundary differences of delineation based on anatomical landmarks and palpation marks.

\begin{tabular}{lcccc}
\hline Boundary & Mean, $\mathrm{mm}$ & Maximum, $\mathrm{mm}$ & Minimum, $\mathrm{mm}$ & Coefficient of variation \\
\hline Medial & 3.35 & 18.0 & -9.2 & 2.16 \\
Lateral & 5.57 & 20.4 & -7.2 & 2.22 \\
Cephalic & 1.75 & 18.0 & -21.0 & 6.64 \\
Caudal & 11.25 & 21.0 & 6.0 & 0.36 \\
\hline
\end{tabular}

Table III. Correlation between target volumes and boundaries delineated based on anatomical landmarks and palpation marks.

\begin{tabular}{|c|c|c|c|c|c|c|}
\hline \multirow[b]{2}{*}{ Boundary } & \multicolumn{2}{|l|}{ CTVan } & \multicolumn{2}{|l|}{ CTVpa } & \multicolumn{2}{|l|}{ CTVgl } \\
\hline & Correlation factor (r) & P-value & Correlation factor (r) & P-value & Correlation factor (r) & P-value \\
\hline Medial & -0.549 & 0.064 & -0.681 & 0.015 & -0.285 & 0.370 \\
\hline Lateral & -0.252 & 0.430 & -0.460 & 0.132 & -0.243 & 0.446 \\
\hline Cephalic & -0.601 & 0.051 & -0.748 & 0.005 & -0.542 & 0.069 \\
\hline Caudal & -0.143 & 0.658 & -0.319 & 0.313 & -0.311 & 0.326 \\
\hline
\end{tabular}

CT, computed tomography; CTVgl, clinical target volume by CT images; CTVpa, clinical target volume by breast palpation; CTVan, clinical target volume by anatomical landmarks.

in CTVgl was $0.264 \pm 0.109$ (range, $0.130-0.423$ ), the DI of CTVgl in CTVpa was 0.989 \pm 0.008 (range, 0.976-1.000) and the DI of CTVpa in CTVgl was 0.329 \pm 0.115 (range, 0.151-0.552).

The boundary defined by CTVan was regarded as the standard. The distance between CTVpa and CTVan with regard to the boundaries was measured in the medial, lateral, cephalic and caudal directions (CTVpa boundary located outside the CTVan boundary was depicted as a negative value; otherwise it was depicted as a positive value), and their mean values were $3.35 \pm 7.23,5.57 \pm 13.37,1.75 \pm 11.62$ and $11.25 \pm 4.07 \mathrm{~mm}$, respectively. The boundary differences and the correlation between target volumes and boundaries delineated are listed in detail in Tables II and III.

\section{Discussion}

Bentel et al (11) highlighted the fact that the glandular breast tissue reflected by X-ray images cannot represent the real volume of the breast, particularly at the superior and lateral quadrants, and axillary tail; however, around $60 \%$ of cancer occurs in these areas (17). In the current study, the delineated CTVgl boundary based on the images of the CT scan was significantly smaller than that delineated by the other two methods; the target volume was only 24.7 and $31.7 \%$ of that estimated by CTVan and CTVpa, respectively. One possible reason for this may be that $\mathrm{CTVgl}$ included only the dense glandular breast tissue and large breast ducts; the end of acinus and small breast ducts may be excluded as they cannot be distinguished from the adipose tissue by X-ray images. Therefore, there is a high risk of missing the target volume if the analysis is based only on the images of the CT scan. In addition, the findings of the current study indicated that the target volume defined by CTVan and CTVpa could contain 99\% of the target volume defined by CTVgl. The remaining
$1 \%$ target volume was revealed as scattered tiny cloudy shadows on the images of the CT scan, which exceeded the $5-\mathrm{mm}$ thickness of the subcutaneous tissue. According to anatomical studies, the thickness of the subcutaneous adipose tissue of the breast is only 0.5 to $2.5 \mathrm{~mm}$ (18-20), therefore, the anterior boundary of target volume delineation is usually set at a thickness of 3 to $5 \mathrm{~mm}$ of the subcutaneous tissue to ensure sufficient irradiation without excessive exposure of normal skin and subcutaneous tissue.

The results of the current study found that the target volume defined by CTVpa was $22 \%$ smaller than that defined by CTVan, and the CI of the two methods was 0.644 (range, 0.490-0.859). Further comparison indicated that the boundary defined by CTVpa was within that defined by CTVan, however, there was a large variation among patients; the most significant difference was at the cephalic boundary with a coefficient of variation up to 6.64 , followed by the caudal and lateral boundaries with a coefficient of variation of 0.36 , consistent with the values published in the literature. Giezen et al (21) found that the target volume based on the images of the magnetic resonance imaging (MRI) scan was $4 \%$ larger than that defined by the images of the CT scan; a significant difference was found in the lateral and medial superior directions, with a $17 \%$ alteration in the target center toward the cephalic direction and a $3 \%$ alteration in the target center toward the dorsal direction. By contrast, Hurkmans et al (12) found that the target volume varied differently by each delineation, and a significant difference was found in the posterior of the breast, and the cephalic and medial boundaries, with a difference of 42, 28 and $24 \mathrm{~mm}$, respectively; the difference was relatively smaller at the anterior, caudal and lateral boundaries, with values of 6,15 and $8 \mathrm{~mm}$, respectively. The results of the current study also found that the difference in cephalic and medial boundaries was negatively correlated with the target 
volume defined by CTVpa, i.e., the larger the target volume of CTVpa, the smaller the boundary difference. Occasionally, the breast volume may exceed the boundary defined by CTVan; however, the lateral or caudal boundary difference was not significantly correlated with target volumes defined by either method. This is due to a lack of clear boundary between the glandular breast tissue and non-breast tissue on the images of the CT scan. The caudal and lateral boundaries are relatively clear due to natural folds or drooping of the breast tissue, therefore, delineation at the medial or cephalic boundary is relatively arbitrary.

Given the limitation of the CT scan images to define the boundary of the glandular breast tissue, the delineator and delineation methods (or strategy) are important for defining the CTV. For instance, Struikmans et al (10) reported that five delineators were used in delineating CTV based on the CT scan images without using other criteria; the CTV finally ranged between 229 and 1,214 cc, with a significant difference. By contrast, Xu et al (22) found that there was no significant difference among the five delineators with regard to CTV with same delineation criteria, even when there was a clear difference prior to the criteria being standardized among them. The majority of the delineators have been adjusted significantly with regard to CTV according to the delineation criteria. Therefore, the standardized delineation criteria could significantly reduce the variation among patients and delineators regarding CTV delineation, which is in agreement with the results of the study by Wang et al (23), which reported that the variation in CTV delineated by one individual was not affected by the CT scanning mode if standardized criteria were used. Metal markers on the tumor bed, the tumor bed location and the age of the patient are significant factors in CTV delineation. If the location of the tumor bed is inclined too much on one side, subjective judgment of the delineator will affect the consistency of delineation (24). Adipose content of glandular breast tissue is positively correlated with age, and as judged by the images of the CT scan; the higher the adipose tissue content, the higher the difficulty in delineating the target volume. Therefore, palpation markers may be more valuable in elderly patients.

Hurkmans et al (12) suggested that the metal wires placed around the breast could reduce the arbitrary variation among delineators, however, accurate delineation depends on the pathological knowledge of the delineator on glandular breast tissue and information provided by advanced imaging techniques. MRI has a higher resolution on adipose tissue, and is able to reveal the structure and surrounding soft tissue of the breast more clearly. The major type of tissue in the cephalic direction of the breast is the adipose tissue, which supports glandular breast tissue; therefore, the volume delineated by the MRI scan is larger than that delineated by the CT scan. Hence, MRI scan images will be of use in target volume delineation, and the fusion of images from the CT and MRI scans has already been widely used in target delineation for brain tumors, nasopharyngeal carcinoma and prostate cancer (25-27). Due to the profound difference in the posture of each patient, there are significant variations between the MRI and CT scan images of the breast; however, the fusion of the images of the MRI and CT scans is less feasible and is difficult for target volume delineation of the breast, therefore, this technique remains to be improved and investigated further. By contrast, the breast parenchyma are wrapped by the surrounding fibrous connective tissues and adipose tissues, which function as supportive structures, resulting in the difficulty to clearly distinguish the glandular breast tissue even by MRI scan.

In summary, due to the limitation with regard to the glandular breast tissue, it is not rational if the target volume delineation is based only on the CT scan images, as this will lead to missing target volume. CT scans in combination with MRI would aid in defining the target volume, but requires further investigation and improvements to the stabilization of the posture of the patients and image fusion. Currently, a combination of palpation marks and anatomical landmarks to define the contouring scope of the breast is suggested to as a relatively rational method for delineating the target volume of the breast. It is worth noting that the knowledge and skill of the delineators is important for accurate delineation, and the size and texture of the breast may affect the determination of the breast boundaries by palpation, particularly the cephalic and medial boundaries.

\section{References}

1. Liljegren G, Holmberg L, Bergh J, Lindgren A, Tabár L, Nordgren $\mathrm{H}$ and Adami HO: 10-year results after sector resection with or without postoperative radiotherapy for stage I breast cancer: a randomized trial. J Clin Oncol 17: 2326-2333, 1999.

2. Fisher B, Anderson S, Bryant J, Margolese RG, Deutsch M, Fisher ER, et al: Twenty-year follow-up of a randomized trial comparing total mastectomy, lumpectomy, and lumpectomy plus irradiation for the treatment of invasive breast cancer. N Engl J Med 347: 1233-1241, 2002.

3. No authors listed: Favourable and unfavourable effects on long-term survival of radiotherapy for early breast cancer: an overview of the randomised trials. Early Breast Cancer Trialists' Collaborative Group. Lancet 355: 1757-1770, 2000.

4. Early Breast Cancer Trialists' Collaborative Group (EBCTCG); Darby S, McGale P, Correa C, et al: Effect of radiotherapy after breast-conserving surgery on 10-year recurrence and 15-year breast cancer death: meta-analysis of individual patient data for 10,801 women in 17 randomised trials. Lancet 378: 1707-1716, 2011.

5. Sedlmayer F, Sautter-Bihl ML, Budach W, et al; Breast Cancer Expert Panel of the German Society of Radiation Oncology (DEGRO): DEGRO practical guidelines: radiotherapy of breast cancer I: radiotherapy following breast conserving therapy for invasive breast cancer. Strahlenther Onkol 189: 825-833, 2013.

6. Hughes KS, Schnaper LA, Bellon JR, et al: Lumpectomy plus tamoxifen with or without irradiation in women age 70 years or older with early breast cancer: long-term follow-up of CALGB 9343. J Clin Oncol 31: 2382-2387, 2013.

7. Beitsch PD, Shaitelman SF and Vicini FA: Accelerated partial breast irradiation. J Surg Oncol 103: 362-368, 2011.

8. Croog VJ, Wu AJ, McCormick B and Beal KP: Accelerated whole breast irradiation with intensity-modulated radiotherapy to the prone breast. Int J Radiat Oncol Biol Phys 73: 88-93, 2009.

9. Mouw KW and Harris JR: Irradiation in early-stage breast cancer: conventional whole-breast, accelerated partial-breast, and accelerated whole-breast strategies compared. Oncology (Williston Park) 26: 820-830, 2012.

10. Struikmans H, Wárlám-Rodenhuis C, Stam T, Stapper G, Tersteeg RJ, Bol GH and Raaijmakers CP: Interobserver variability of clinical target volume delineation of glandular breast tissue and of boost volume in tangential breast irradiation. Radiother Oncol 76: 293-299, 2005.

11. Bentel GC, Marks LB, Hardenbergh PH and Prosnitz L: Variability of the location of internal mammary vessels and glandular breast tissue in breast cancer patients undergoing routine CT-based treatment planning. Int J Radiat Oncol Biol Phys 44: 1017-1025, 1999. 
12. Hurkmans CW, Borger JH, Pieters BR, Russell NS, Jansen EP and Mijnheer BJ: Variability in target volume delineation on CT scans of the breast. Int J Radiat Oncol Biol Phys 50: 1366-1372, 2001

13. Kraus-Tiefenbacher U, Sfintizky A, Welzel G, Simeonova A, Sperk $\mathrm{E}$, Siebenlist K, et al: Factors of influence on acute skin toxicity of breast cancer patients treated with standard three-dimensional conformal radiotherapy (3D-CRT) after breast conserving surgery (BCS). Radiat Oncol 7: 217, 2012.

14. Barnett GC, Wilkinson JS, Moody AM, Wilson CB, Twyman N, Wishart GC, et al: The Cambridge Breast Intensity-modulated Radiotherapy Trial: patient- and treatment-related factors that influence late toxicity. Clin Oncol (R Coll Radiol) 23: 662-673, 2011.

15. Hof H, Rhein B, Haering P, Kopp-Schneider A, Debus J and Herfarth K: 4D-CT-based target volume definition in stereotactic radiotherapy of lung tumours: comparison with a conventional technique using individual margins. Radiother Oncol 93: 419-423, 2009.

16. Ezhil M, Vedam S, Balter P, Choi B , Mirkovic D , Starkschall G and Chang JY: Determination of patient-specific internal gross tumor volumes for lung cancer using four-dimensional computed tomography. Radiat Oncol 4: 4, 2009.

17. Sohn VY, Arthurs ZM, Sebesta JA and Brown TA: Primary tumor location impacts breast cancer survival. Am J Surg 195: 641-644, 2008.

18. Na K, Xiang K and Wang G: Assessment of thickness for breast skin and gland among office lady by ultrasound. Zhonghua Xian Dai Ying Xiang Xue Za Zhi 1: 12-14, 2004 (In Chinese).

19. Wang XS and Liao KH (eds): Yang Guoliang Dermatology. 12 th edition. Shanghai Scientific and Technological Literature Publishing House. Shanghai, 2005 (In Chinese).

20. Li HD, Cai GB, Wang YQ, Zhang R, Li BB, Li TY, et al: Study of normal human skin with $50 \mathrm{MHz}$ ultrasound biomicroscope. Zhong Guo Yi Xue Ying Xiang Ji Shu 24: 751-753, 2008 (In Chinese).
21. Giezen M, Kouwenhoven E, Scholten AN, Coerkamp EG, Heijenbrok M, Jansen WP, et al: Magnetic resonance imaging- versus computed tomography-based target volume delineation of the glandular breast tissue (clinical target volume breast) in breast-conserving therapy: an exploratory study. Int J Radiat Oncol Biol Phys 81: 804-811, 2011.

22. Xu M, Li J, Yu Z, Yang T, Wang X, Zhou X, et al: Effect of target delineation standard training for radiotherapy on breast cancer after breast conserving surgery. Zhonghua Fang She Zhong Liu Xu Za Zhi 21: 534-537, 2012 (In Chinese).

23. Wang S, Li J, Zhang Y, Wang W, Li F, Xu M, et al: Comparative study of 3D-CT and 4D-CT target delineation of breast clinic volume for radiotherapy after breast conserving surgery. Zhonghua Ru Xian Bing Za Zhi 6: 494-503, 2012 (In Chinese)

24. Huang XB, Chen JY and Jiang GL: Factors influencing clinical target volume delineation of intact breast in intensity-modulated radiotherapy for breast cancer. Ai Zheng 25: 62-65, 2006 (In Chinese).

25. Khoo VS, Dearnaley DP, Finnigan DJ, Padhani A, Tanner SF and Leach MO: Magnetic resonance imaging (MRI): considerations and applications in radiotherapy treatment planning. Radiother Oncol 42: 1-15, 1997.

26. Emami B, Sethi A and Petruzzelli GJ: Influence of MRI on target volume delineation and IMRT planning in nasopharyngeal carcinoma. Int J Radiat Oncol Biol Phys 57: 481-488, 2003.

27. Manavis J, Sivridis L and Koukourakis MI: Nasopharyngeal carcinoma: the impact of CT-scan and of MRI on staging, radiotherapy treatment planning, and outcome of the disease. Clin Imaging 29: 128-133, 2005. 